\title{
Corrigendum: Normal High HbA1c a Risk Factor for Abnormal Pain Threshold in the Japanese Population
}

\begin{abstract}
Chieko Itabashi ${ }^{1}$, Hiroki Mizukami i ${ }^{1 *}$, Sho Osonoi ${ }^{1}$, Kazuhisa Takahashi ${ }^{1}$, Kazuhiro Kudo ${ }^{1}$, Kanichiro Wada ${ }^{2}$, Wataru Inaba ${ }^{1}$, Guo Danyang ${ }^{1}$, Chiaki Uchida ${ }^{1,3}$, Satoko Umetsu ${ }^{1,3}$, Akiko Igawa ${ }^{1,3}$, Saori Ogasawara ${ }^{1}$, Masaki Ryuzaki ${ }^{1}$, Kouji Komeda ${ }^{1}$, Yasuyuki Ishibashi ${ }^{2}$, Soroku Yagihashi ${ }^{1}$ and Shigeyuki Nakaji ${ }^{4}$

${ }^{1}$ Department of Pathology and Molecular Medicine, Hirosaki University Graduate School of Medicine, Hirosaki, Japan, ${ }^{2}$ Department of Orthopedic Surgery, Hirosaki University Graduate School of Medicine, Hirosaki, Japan, ${ }^{3}$ Department of Gastrointestinal Surgery, Hirosaki University Graduate School of Medicine, Hirosaki, Japan, ${ }^{4}$ Department of Social Medicine, Hirosaki University Graduate School of Medicine, Hirosaki, Japan
\end{abstract}

Keywords: small fiber dysfunction, diabetic polyneuropathy, HbA1c, painful, small fiber assessment

\section{A Corrigendum on}

Normal High HbAlc a Risk Factor for Abnormal Pain Threshold in the Japanese Population by Itabashi, C., Mizukami, H., Osonoi, S., Takahashi, K., Kudo, K., Wada, K., et al. (2019). Front. Endocrinol. 10:651. doi: 10.3389/fendo.2019.00651

\section{OPEN ACCESS}

Edited and reviewed by: Jan Polák,

Charles University, Czechia

*Correspondence:

Hiroki Mizukami hirokim@hirosaki-u.ac.jp

Specialty section: This article was submitted to Clinical Diabetes,

a section of the journal Frontiers in Endocrinology

Received: 02 January 2020 Accepted: 26 February 2020 Published: 10 March 2020

Citation:

Itabashi C, Mizukami H, Osonoi S, Takahashi K, Kudo K, Wada K, Inaba W, Danyang G, Uchida C, Umetsu S, Igawa A, Ogasawara S, Ryuzaki M, Komeda K, Ishibashi Y, Yagihashi S and Nakaji S (2020)

Corrigendum: Normal High HbA1c a

Risk Factor for Abnormal Pain

Threshold in the Japanese Population.

Front. Endocrinol. 11:130.

doi: 10.3389/fendo.2020.00130
In the original article, old type of electrodes were incorrectly identified as (NM-990W) instead of (NM-983W). In addition, the average of P-IES in non-diabetic/IFG subjects was incorrect. The correct value is " $0.15 \pm 0.01$."

A correction has been made in the following places:

The Material and Methods section, subsection P-IES Measurement, paragraph 1:

"For nociceptive stimulation, an IES method was adopted using a disposable concentric bipolar needle electrode (NM-983W; Nihon Kohden Corp., Tokyo, Japan) which was connected to a specific stimulator for cutaneous $\mathrm{A} \delta$ and C fibers as previously described (PNS-7000; Nihon Kohden) (15)."

The Abstract, subsection Results:

"P-IES was elevated with increasing of age in women but not in men. Average P-IES (mA) was increased in IFG subjects $(n=55,0.20 \pm 0.03)$ compared with normoglycemic/non-IFG individuals $(n=894,0.15 \pm 0.01)(p<0.01)$. It was comparable between IFG and a group of normal high $\mathrm{HbAlc}$ (5.9-6.4\%). Univariate linear regression analyses showed no influence of sex, triglyceride, or cholesterol on the value of P-IES. In contrast, there were significant correlations between P-IES and serum HbA1c level $(\beta=0.120, p<0.001)$ Adjustments for the multiple clinical measurements confirmed positive correlation of P-IES with HbA1c $(\beta=0.077, p=0.046)$."

The authors apologize for this error and state that this does not change the scientific conclusions of the article in any way. The original article has been updated.

Copyright $\odot 2020$ Itabashi, Mizukami, Osonoi, Takahashi, Kudo, Wada, Inaba, Danyang, Uchida, Umetsu, Igawa, Ogasawara, Ryuzaki, Komeda, Ishibashi, Yagihashi and Nakaji. This is an open-access article distributed under the terms of the Creative Commons Attribution License (CC BY). The use, distribution or reproduction in other forums is permitted, provided the original author(s) and the copyright owner(s) are credited and that the original publication in this journal is cited, in accordance with accepted academic practice. No use, distribution or reproduction is permitted which does not comply with these terms. 